\title{
SUPER AND SUBSOLUTIONS FOR ELLIPTIC EQUATIONS ON ALL OF $\mathbb{R}^{n}$
}

\section{G. A. AFROUZI and H. GHASEMZADEH}

Received 26 October 2001

By construction sub and supersolutions for the following semilinear elliptic equation $-\triangle u(x)=\lambda g(x) f(u(x)), x \in \mathbb{R}^{n}$, which arises in population genetics, we derive some results about the theory of existence of solutions as well as asymptotic properties of the solutions for every $n$ and for the function $g: \mathbb{R}^{n} \rightarrow \mathbb{R}$ such that $g$ is smooth and is negative at infinity.

2000 Mathematics Subject Classification: 35J60.

1. Introduction. In this paper, we discuss the existence and nonexistence of solutions as well as asymptotic properties of the solutions of the equation

$$
-\triangle u(x)=\lambda g(x) f(u(x)), \quad x \in \mathbb{R}^{n}, 0 \leq u(x) \leq 1
$$

which arises in population genetics (see $[7,11])$. The unknown function $u$ corresponds to the relative frequency of an allele and is hence constrained to have values between 0 and 1 . The real parameter $\lambda>0$ corresponds to the reciprocal of a diffusion coefficient.

We assume throughout that $g: \mathbb{R}^{n} \rightarrow \mathbb{R}$ is smooth which changes sign on $\mathbb{R}^{n}$. Also we will assume throughout that $f$ satisfies the condition $f:[0,1] \rightarrow \mathbb{R}$ is a smooth function such that $f(0)=f(1)=0, f^{\prime}(0)>0, f^{\prime}(1)<0$, and $f(u)>0$ for all $0<u<1$.

By the definition of $f$, it is clear that (1.1) has the trivial solutions $u \equiv 0$ and $u \equiv 1$.

The existence of solutions for (1.1) in the bounded region case with Dirichlet or Neumann boundary conditions is discussed in [7,11], but in this case all of $\mathbb{R}^{n}$ is much more complicated (see [3, 6, 7, 8, 9, 12, 13]). The results obtained in [7] with the assumption that $g$ is negative at infinity show that the existence theory for solutions of (1.1) is very different for the two cases $n=1,2$ and $n \geq 3$.

Some of the nontrivial solutions were bifurcating off the trivial solution $u \equiv 0$. In order to investigate these bifurcation phenomena, it was necessary to understand the eigenvalues and eigenfunctions of the corresponding linearized problem

$$
-\triangle u(x)=\lambda g(x) f^{\prime}(0) u(x), \quad x \in \mathbb{R}^{n} .
$$

The existence of positive principal eigenfunctions of (1.2) with the following conditions on $g$ was considered in [6]:

(i) $g$ is negative and bounded away from zero at infinity; or

(ii) $|g(x)| \leq k /\left(1+|x|^{2}\right)^{\alpha}, n \geq 3$,

for some constants $k>0$ and $\alpha>1$, and these results for the case $g^{+} \in L^{n / 2}\left(\mathbb{R}^{n}\right)$, $n \geq 3$ where $g^{+}(x)=\max \{g(x), 0\}$ are extended in [3]. 
In this paper, we investigate the existence of solutions of (1.1) with the assumption that $g$ or $g^{+}$are small at infinity.

Our analysis is based on the construction of sub and supersolutions.

It is proved in [2] that the positive principal eigenvalue of the Dirichlet boundary value problem

$$
\begin{aligned}
-\triangle u(x) & =\lambda g(x) u(x), \quad x \in D, \\
u(x) & =0, \quad x \in \partial D,
\end{aligned}
$$

where $D$ is a bounded domain with smooth boundary has the variational characterisation

$$
\lambda_{1}^{+}(D)=\inf \left\{\int_{D}|\nabla u(x)|^{2} d x: u \in H_{0}^{1}(D), \int_{D} g u^{2} d x=1\right\} .
$$

Also, it is well known that the above infimum is attained and a minimizer $\phi_{1}>0$ is smooth, that is, $c^{2}(\bar{D})$. Hence $\phi_{1}$ satisfies the Dirichlet boundary value problem (1.3), so $\phi_{1}$ is a principal eigenfunction corresponding to principal eigenvalue $\lambda_{1}^{+}(D)$.

Suppose, however, that $g=g^{+}-g^{-}$where $g^{+}(x)=\max \{g(x), 0\}$ and $g^{-}(x)=$ $\min \{g(x), 0\}$.

If $n \geq 3$ and $g^{+} \in L^{n / 2}\left(\mathbb{R}^{n}\right)$, then for all $u \in H_{0}^{1}(D)$ such that $\int_{D} g u^{2} d x=1$ we have

$$
\begin{aligned}
1=\int_{D} g u^{2} d x & \leq \int_{D} g^{+} u^{2} d x \\
& \leq\left\|g^{+}\right\|_{L^{n / 2}(D)}\|u\|_{L^{2 n /(n-2)}(D)}^{2} \\
& \leq c(n)\left\|g^{+}\right\|_{L^{n / 2}(D)}\|\nabla u\|_{L^{2}(D)}^{2}
\end{aligned}
$$

where $c(n)$ is the embedding constant of $H_{0}^{1}(D)$ into $L^{2 n /(n-2)}(D)$ and is independent of $D$ (see Brézis and Nirenberg [5, page 443]). Thus

$$
\lambda_{1}^{+}(D) \geq\|\nabla u\|_{L^{2}(D)}^{2} \geq\left\{c(n)\left\|g^{+}\right\|_{L^{n / 2}(D)}\right\}^{-1}>0 .
$$

Also, it is well known (see [1]) that if $g^{+} \in L^{n / 2}\left(\mathbb{R}^{n}\right)$, then $\lambda^{*}=\lim _{\mathbb{R} \rightarrow \infty} \lambda_{1}^{+}\left(B_{R}(0)\right)$ exists and $\lambda^{*}$ is the principal eigenvalue of the equation

$$
-\triangle u(x)=\lambda g(x) u(x), \quad x \in \mathbb{R}^{n}
$$

and there exists a corresponding principal eigenfunction $\phi$ such that $\phi(x) \rightarrow 0$ as $|x| \rightarrow \infty$. In addition, $\lambda^{*}$ can be characterized as follows (see [1, Lemma 2.7])

$$
\lambda^{*}=\inf \left\{\int_{\mathbb{R}^{n}}|\nabla u(x)|^{2} d x: u \in c_{0}^{\infty}\left(\mathbb{R}^{n}\right), \int_{\mathbb{R}^{n}} g u^{2} d x=1\right\}
$$


THEOREM 1.1 (see [10]). If $\lambda>\lambda^{*}$, then there exists $\underline{u} \geq 0(\underline{u} \neq 0)$ with compact support such that $\underline{u}$ is a subsolution of

$$
\begin{aligned}
-\triangle u(x) & =\lambda g(x) f(u(x)), \quad x \in B_{R}(0), \\
u(x) & =0, \quad x \in \partial B_{R}(0)
\end{aligned}
$$

for all $\mathbb{R}$ sufficiently large, also we can choose $\underline{u}$ sufficiently small.

2. Sub and supersolutions for $n \geq 3$. We assume $D \subset \mathbb{R}^{n}$ is a bounded region with smooth boundary. We consider the following boundary value problem:

$$
\begin{aligned}
-\triangle u(x) & =\lambda g(x) f(u(x)), \quad x \in D, \\
u(x) & =0, \quad x \in \partial D .
\end{aligned}
$$

If $\lambda>0$ be fixed, we can choose $c>0$ such that for $u, 0 \leq u \leq 1$, the function $u \rightarrow$ $\lambda g(x) f(u)+c u$, for every $x \in D$, is an increasing function.

Let $h(x, u)=\lambda g(x) f(u)+c u$, then we have $h(x, 0) \equiv 0$ and $h(x, 1) \equiv c$. We can write (2.1) as

$$
\begin{aligned}
-\triangle u(x)+c u(x) & =h(x, u(x)), \quad x \in D, \\
u(x) & =0, \quad x \in \partial D .
\end{aligned}
$$

It is well known that (2.2) has a unique solution $u=K f$ (see Amann [4]), where $K$ is given by an integral operator whose kernel is the Green's function for the problem, that is,

$$
(K f)(x)=\int_{D} G(x, y) h(y, u(y)) d y .
$$

In (2.3), $G(x, y)$ is the Green's function of the operator $-\triangle+c$ with Dirichlet boundary condition, also we can write (2.3) as $u=K N(u)$ in where $K: c(\bar{D}) \rightarrow c^{\alpha}(\bar{D})$ is a compact linear integral operator with kernel $G$ (see [4]) and $N: c(\bar{D}) \rightarrow c(\bar{D})$ is the Nemytskii operator corresponding to $h$. Since $h(x, \cdot)$ is increasing, it is easy to see that $N$ is an increasing operator, that is, if $u_{1} \geq u_{2}$, then $N u_{1} \geq N u_{2}$.

We call $u \in c^{2}(D)$ is a subsolution of (2.2) or equivalently (2.1) if we have

$$
\begin{aligned}
-\triangle u(x)+c u(x) & \leq h(x, u(x)), \quad x \in D, \\
u(x) & \leq 0, \quad x \in \partial D,
\end{aligned}
$$

and $u \in c(\bar{D})$ is a subsolution of (2.3) if

$$
u(x) \leq \int_{D} G(x, y) h(y, u(y)) d y, \quad x \in D,
$$

that is, $u \leq K N(u)$. The definition of supersolution is quite similar.

It is well known that if $v, w$ are sub and supersolutions of (2.2) (or for (2.3)), respectively, and $v \leq w$, then there exists a solution $u$ of (2.2) (of (2.3)) such that $v \leq u \leq w$. 
3. The case when $n=1,2$. In this section, we consider the problem

$$
\begin{gathered}
-\triangle u(x)=\lambda g(x) f(u(x)), \quad x \in \mathbb{R}^{n}, \\
0 \leq u(x) \leq 1, \quad x \in \mathbb{R}^{n},
\end{gathered}
$$

where $g: \mathbb{R}^{n} \rightarrow \mathbb{R}$ is a continuous function which changes sign on $\mathbb{R}^{n}$ and it has the following condition: $(\mathrm{G})$ there exists $R_{0}>0$ such that $g(x)<0$ for all of $x \in \mathbb{R}^{n}$, whenever $|x|>R_{0}$.

Also $f \in c^{1}([0,1])$ with the conditions

$$
f(0)=0=f(1), \quad f^{\prime}(0)>0, \quad f^{\prime}(1)<0, \quad f(u)>0, \quad 0<u<1 .
$$

THEOREM 3.1 (see [7]). Let $u$ be a nontrivial solution of (4.1). Then there exists a real constant $k$ such that $0<u(x)<k<1$ for all of $x$ in $\mathbb{R}^{n}$.

Now by using Theorem 3.1 and condition $(G)$ on $g$, we conclude that

$$
\triangle u(x)>0
$$

for all of $x \in \mathbb{R}^{n}$ with $|x|>R_{0}$.

THEOREM 3.2. Let $u$ be a nontrivial solution of (4.1). Then $u$ is nonconstant in out of the ball $B_{R_{0}}(0)$.

Proof. Using assumption on $g$, we have $\triangle u(x)>0$ for all of $x \in \mathbb{R}^{n}$ with $|x|>R_{0}$, so $|\nabla u(x)|>0$ whenever $|x|>R_{0}$. Hence $u$ is a nonconstant function in out of the ball $B_{R_{0}}(0)$.

THEOREM 3.3. Let $n=1$ and $u$ be a nontrivial solution of (4.1). Then $u$ is a strictly decreasing function on $\left(R_{0}, \infty\right)$ and increasing function on $\left(-\infty,-R_{0}\right)$.

Proof. By using assumption on $g$, we have $u^{\prime \prime}(x)>0$ for all of $x \in \mathbb{R}^{n}$ with $|x|>$ $R_{0}$. So, $u$ can have only one of the possibilities (a) and (b) in Figure 3.1.

Figure 3.1(a) is impossible because we must have $0 \leq u(x) \leq 1$ for all $x \in \mathbb{R}^{n}$. So $u$ satisfy in Figure 3.1(b), thus $u$ is strictly decreasing in out of ball $B_{R_{0}}(0)$.

THEOREM 3.4. Let $n=2$ and $u$ be a solution of (4.1) which is radially symmetric, then $u$ is a strictly monotone function in out of the ball $B_{R_{1}}(0)$, where $R_{1}>R_{0}$.

Proof. It is obvious by using maximum principle.

4. The case when $n \geq 3$. Let $g$ satisfy condition (G). It is easy to see that

$$
\bar{u}(x)= \begin{cases}1, & |x| \leq R_{0}, \\ \left(\frac{R_{0}}{|x|}\right)^{(n-2)}, & |x|>R_{0},\end{cases}
$$

is a supersolution of (4.1), so we are ready to prove the following theorem.

THEOREM 4.1. If $\lambda>\lambda^{*}$, then there exists a nonconstant solution $u$ of (4.1) such that

$$
\lim _{|x| \rightarrow \infty} u(x)=0
$$




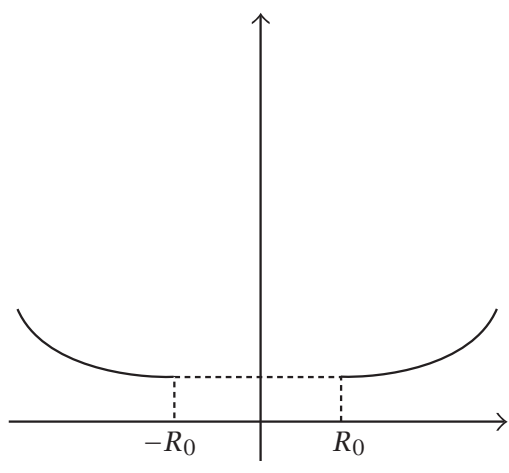

(a)

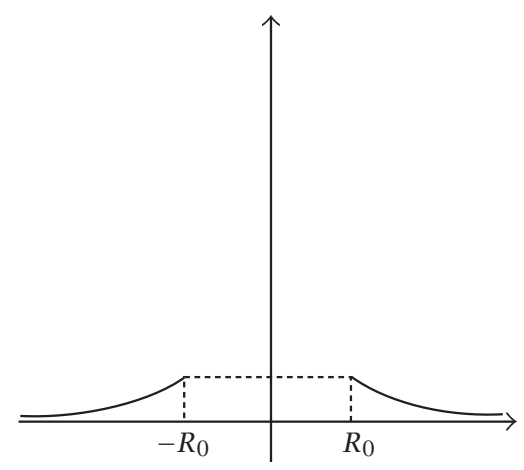

(b)

FIGURE 3.1

Proof. We consider $\bar{u}$ as a supersolution of (4.1). Also there exists a subsolution $\underline{u}$ of (4.1) with compact support and sufficiently small (see [10]). So we can choose $\underline{u}$ such that $\underline{u} \leq \bar{u}$, so there exists a solution $u$ of (4.1) such that $\underline{u} \leq u \leq \bar{u}$. Also by using the definition of $\bar{u}$, we have $\lim _{|x| \rightarrow \infty} u(x)=0$.

THEOREM 4.2. Let $\alpha>1$ and $\lambda>0$ be arbitrary. Then there exists a supersolution $\bar{u}$ of (4.1) such that $|\bar{u}(x)| \leq c|x|^{-\beta}$ for a constant $c>0$, and

$$
\beta= \begin{cases}n-2, & n<2 \alpha, \\ 2 \alpha-2, & n>2 \alpha .\end{cases}
$$

Proof. Using condition (G) of the function $g$, we have

$$
\left|g^{+}(x)\right| \leq \frac{k}{\left(1+|x|^{2}\right)^{\alpha}}
$$

where $k \geq M\left(1+R_{0}^{2}\right)^{\alpha}, M=\max g^{+}(x)$. So using [10, Lemma 4.3], the proof is complete.

\section{REFERENCES}

[1] G. A. Afrouzi, Some problems in elliptic equations involving indefinite weight functions, Ph.D. thesis, Heriot-Watt University, Edinburgh, UK, 1997.

[2] G. A. Afrouzi and K. J. Brown, On principal eigenvalues for boundary value problems with indefinite weight and Robin boundary conditions, Proc. Amer. Math. Soc. 127 (1999), no. 1, 125-130.

[3] W. Allegretto, Principal eigenvalues for indefinite-weight elliptic problems in $\mathbb{R}^{n}$, Proc. Amer. Math. Soc. 116 (1992), no. 3, 701-706.

[4] H. Amann, Fixed point equations and nonlinear eigenvalue problems in ordered Banach spaces, SIAM Rev. 18 (1976), no. 4, 620-709.

[5] H. Brézis and L. Nirenberg, Positive solutions of nonlinear elliptic equations involving critical Sobolev exponents, Comm. Pure Appl. Math. 36 (1983), no. 4, 437-477.

[6] K. J. Brown, C. Cosner, and J. Fleckinger, Principal eigenvalues for problems with indefinite weight function on $\mathbb{R}^{n}$, Proc. Amer. Math. Soc. 109 (1990), no. 1, 147-155. 
[7] K. J. Brown, S. S. Lin, and A. Tertikas, Existence and nonexistence of steady-state solutions for a selection-migration model in population genetics, J. Math. Biol. 27 (1989), no. 1, 91-104.

[8] K. J. Brown and N. M. Stavrakakis, Sub-and supersolutions for semilinear elliptic equations on all of $\mathbb{R}^{n}$, Differential Integral Equations 7 (1994), no. 5-6, 1215-1225.

[9] _ Global bifurcation results for a semilinear elliptic equation on all of $\mathbb{R}^{N}$, Duke Math. J. 85 (1996), no. 1, 77-94.

[10] - On the construction of super and subsolutions for elliptic equations on all of $\mathbb{R}^{N}$, Nonlinear Anal. 32 (1998), no. 1, 87-95.

[11] W. H. Fleming, A selection-migration model in population genetics, J. Math. Biol. 2 (1975), no. 3, 219-233.

[12] J. L. Gámez, Sub- and super-solutions in bifurcation problems, Nonlinear Anal. 28 (1997), no. 4, 625-632.

[13] Z. Jin, Principal eigenvalues with indefinite weight functions, Trans. Amer. Math. Soc. 349 (1997), no. 5, 1945-1959.

G. A. Afrouzi: Department of Mathematics, Faculty of Basic Sciences, MaZandaran UNIVERSITY, BABOLSAR, IRAN

E-mail address: afrouzi@umcc.ac.ir

H. Ghasemzadeh: Department of Mathematics, Faculty of Basic Sciences, MazanDARAN UNIVERSITY, BABOLSAR, IRAN 


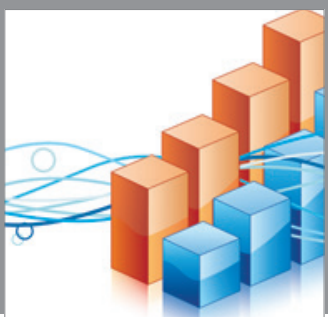

Advances in

Operations Research

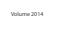

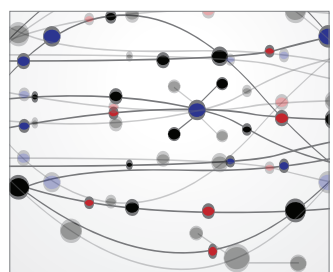

\section{The Scientific} World Journal
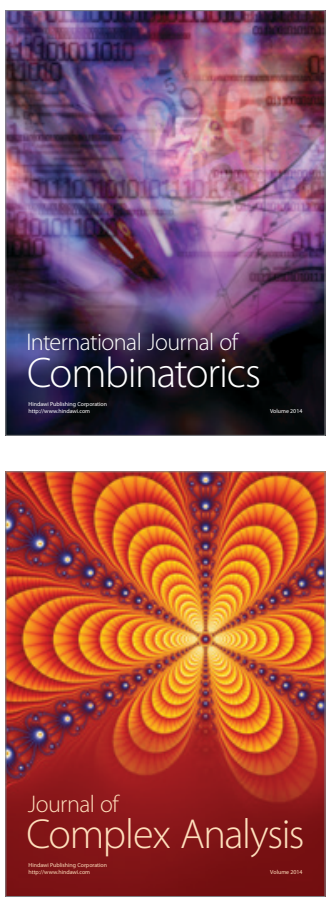

International Journal of

Mathematics and

Mathematical

Sciences
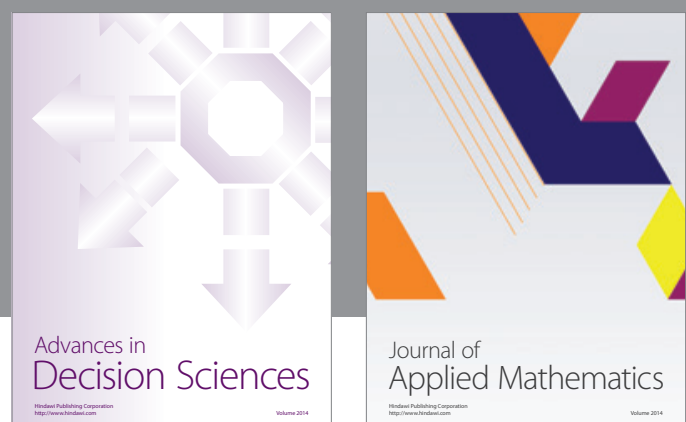

Journal of

Applied Mathematics
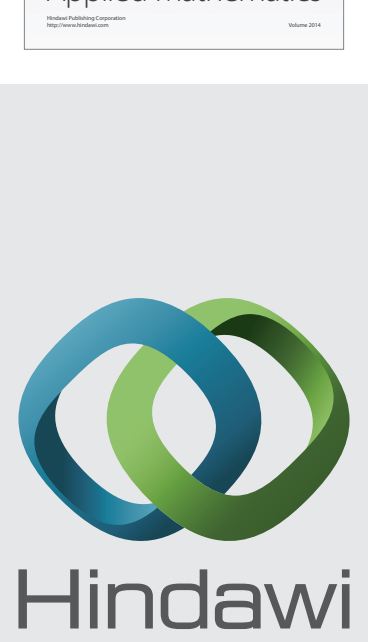

Submit your manuscripts at http://www.hindawi.com
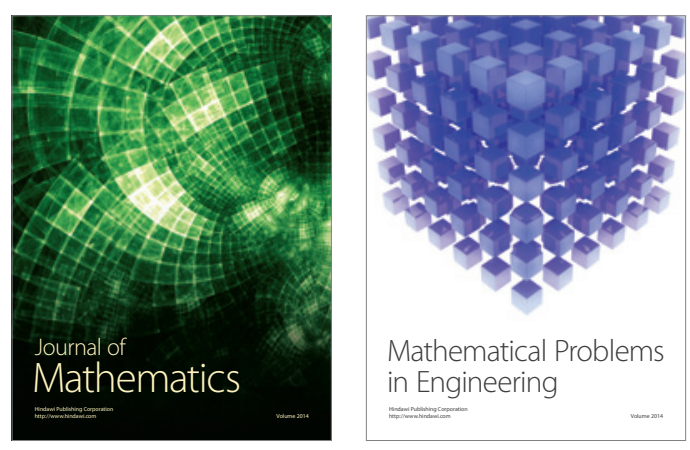

Mathematical Problems in Engineering
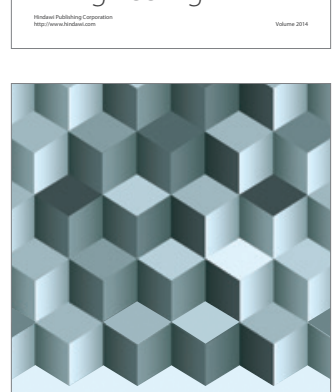

Journal of

Function Spaces
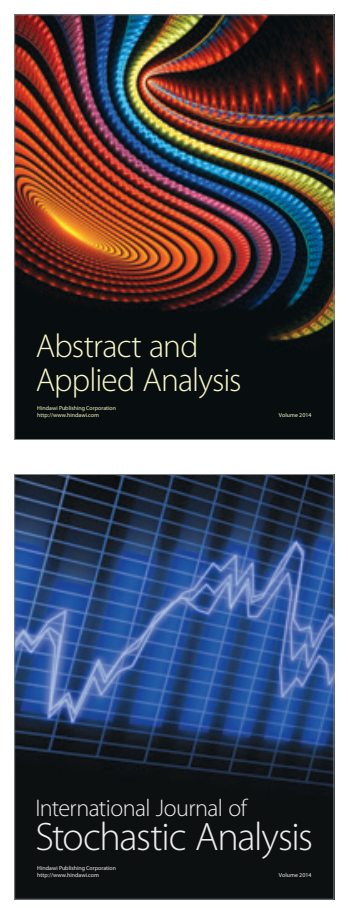

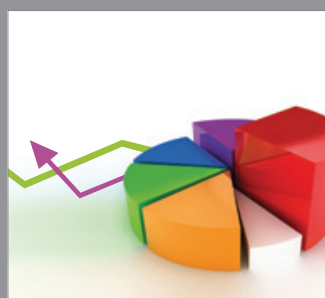

ournal of

Probability and Statistics

Promensencen
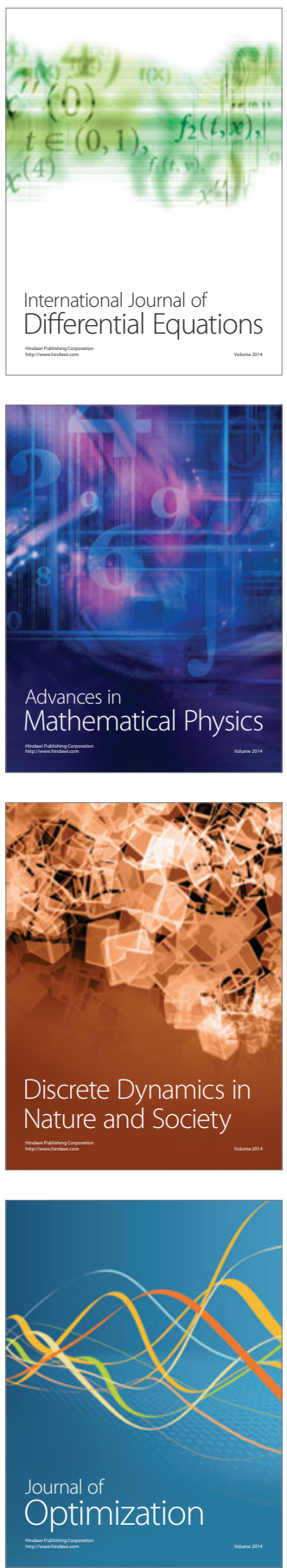